\title{
Optimizing diagnostic imaging through skills mix: costs and opportunities
}

Increasing diagnostic capacity is a national priority to expedite the timeliness and appropriateness of patient treatment interventions. Imaging, encompassing a range of technologies including X-ray, Computer Tomography, Magnetic Resonance Imaging and ultrasound, is a key diagnostic service and central to decision making in most, if not all, disease pathways. However, imaging is an expensive discipline accounting for an estimated 3-5\% of the annual NHS budget. As a result, it is imperative that we maximize service efficiency while optimizing patientoutcomes.

\section{Meeting increasing demand}

Demand for diagnostic imaging examinations has increased annually by up to $10 \%$, depending on modality, for the last 20 years and projected figures anticipate continued growth in demand for the foreseeable future. Nowhere is this more prominent than within the cancer plans for England which call for greater capacity within diagnostic imaging to ensure the ambitious cancer targets are met. But this is just one area where diagnostic imaging is essential to patient diagnosis, treatment and survival. Imaging mustalso meettargets associated with stroke and trauma as well as operating efficiently to manage referrals from other medical specialties. The growth in technological advancements related to molecular imaging and biomarkers suggests that the role of imaging will continue to expand and be central to both diagnostic and preventative health programmes.

Technological advancements in the acquisition of images, combined with extended radiographer working hours, has moderated the impact of increasing demand on throughput targets as actual examination times and patient waiting times have been minimized. However, advancements in imaging technologies have also increased the complexity of the diagnostic examinations which are possible and broadened the disease groups for whom imaging is appropriate. As a result, there is increasing demand for image reporting and longstanding evidence suggests that this demand massively exceeds radiology capacity. As a result, departments have looked at alternative strategies for managing reporting demand, including outsourcing, auto- reporting (non-reporting of certain examinations and referral groups) and delegation of reporting activities to radiographersaspart of a skills mix initiative.

\section{The place for skills mix}

Skills mix was first introduced as part of the NHS modernization agenda towards the end of the last century and promoted blurring of traditional professional boundaries and development of shared skills sets to maximize capacity and improve patient pathways. But within radiology, Swinburne (1971) had already suggested that the radiographer skill set could be expanded to include some tasks traditionally undertaken by radiologists, in particular, defined image reporting tasks. Since then, a large number of studies have been undertaken across different imaging technologies comparing the reporting accuracy of radiographers and consultant radiologists, and significant evidence exists of radiographer competence to report diagnostic images across a range of modalities with a positive financial impact. This was acknowledged in the 2010 spending review which stated:

'We will encourage the NHS to train radiographers to report on more of the straightforward $x$-rays in line with the best practice so that consultant radiologists are free to assess the more complicated images, CT and MRI scans, saving the NHS an estimated $£ 7.9$ million annually (Department of Health, 2010) 
Despite government recognition of the potential of skills mix within diagnostic imaging, adoption has not been consistent nationally. As well as professional anxiety from radiologists with respect to radiographers undertaking activities traditionally seen to be part of their role, anxiety has also been reported within the radiography profession with regard to the delegation of appropriate radiographer tasks to assistant (unqualified) practitioners. The anxieties have been cited as key barriers to maximizing the potential benefits of skills mix. Despite these anxieties, there are a small number of departments that have fully embraced skills mix at all levels to address demand and capacity with reported benefits to service operation.

What is not known is how these departments have developed as positive deviants and overcome the commonly cited barriers to skills mix implementation. Neither has an economic operational assessment of differing models of skills mix implementation been undertaken to determine the relationship between skills mix implementation, service efficiency and deliverables (reporting outcomes that will inform clinical decision making and guide patient treatment).

\section{Conclusions}

As imaging departments nationally are reportedly experiencing a crisis in capacity, with radiography remaining on the UK government 'shortage occupations list' and the Royal college of Radiologists calling for a massive increase in radiologists to address service demands, it is essential that we understand the enablers of service redevelopment and model the impact of different methods of service delivery, and levels of skills mix adoption, on the financial and operational efficiency of imaging departments. If not, then with no reduction in demand envisaged, and with continued need for efficiency savings, image acquisition and reporting targets will not be met, clinical decision making will be poorly informed or delayed and the timely implementation of treatment will be hindered.

Department of Health (2010) Department of Health. Spending Review 2010. http://webarchive. nationalarchives.gov.uk/+/www.dh.gov.uk/en/MediaCentre/Pressreleases/DH_120676 